\title{
XIV. Ventilación mecánica no invasiva en pediatría
}

\author{
RAÚL J. CORRALES V.*
}

\section{Noninvasive ventilation in children}

La ventilación mecánica no invasiva (VNI) en pediatría es un procedimiento de uso creciente. Existen varias enfermedades asociadas a insuficiencia respiratoria aguda o crónica que pueden ser beneficiadas por el apoyo ventilatorio. La ventilación no invasiva puede ser aplicada mediante una interfase oronasal o facial, ocasionando menos daño que la ventilación mecánica invasiva a través de un tubo endotraqueal o traqueostomía. La ventilación mecánica no invasiva es menos utilizada en niños, probablemente debido a que es técnicamente más difícil de aplicar en lactantes y niños pequeños que en adultos.

Los estudios fisiológicos realizados en este grupo etáreo son escasos, no se ha definido el modo ventilatorio óptimo y la programación de los parámetros ventilatorios adecuada para cada condición. Tampoco se han definido los criterios clínicos que justifiquen el empleo de la ventilación mecánica no invasiva en condiciones de falla respiratoria aguda o crónica, ya que la VNI no ha demostrado su eficacia en los diferentes contextos clínicos asociados a falla ventilatoria. Esta debe ser capaz de mejorar, revertir o curar la insuficiencia respiratoria, facilitar el manejo de los enfermos y mejorar su pronóstico vital. Las condiciones básicas del paciente para poder implementar el procedimiento requieren tener algún grado de autonomía respiratoria, escasas secreciones, un mínimo de cooperación y no debe haber obstrucción nasal. Es indispensable disponer de una interface adaptada a la anatomía facial del niño.

\section{Ventilación no invasiva en condiciones agudas}

Aunque no existen trabajos controlados y randomizados que comparen la VNI con la ventilación invasiva, los estudios fisiológicos y ex- periencias clínicas sugieren que la VNI puede ser útil en el manejo de diversas entidades clínicas asociadas a insuficiencia respiratoria aguda en pacientes pediátricos. La ventilación no invasiva con presión positiva continua (CPAP) permite reducir el trabajo ventilatorio y mejorar la ventilación alveolar en la bronquiolitis aguda viral y en la obstrucción de la vía aérea superior $^{1-3}$. La aplicación de CPAP puede reducir las apneas y a su vez disminuir la necesidad de intubación en lactantes con bronquiolitis. La VNI, en especial la presión de soporte y ventilación a presión positiva en dos niveles (BiPAP), está indicada como primera opción durante la descompensación respiratoria aguda o crónica en pacientes con enfermedades neuromusculares o fibrosis quística-7.

La necesidad de VNI durante una descompensación aguda en pacientes con enfermedades neuromusculares puede poner en evidencia la necesidad de utilizar este procedimiento en el ámbito ambulatorio (VNI domiciliaria) ${ }^{8}$. En varios estudios clínicos se ha examinado la eficacia y beneficio clínico de la VNI durante las exacerbaciones infecciosas en pacientes con fibrosis quística, y es considerada la primera opción terapéutica en esta condición.

La VNI representa, así mismo, la primera opción terapéutica en niños con insuficiencia respiratoria aguda hipoxémica. En 28 niños, edad promedio 8 años, con insuficiencia respiratoria aguda hipoxémica principalmente secundaria a neumonía, hospitalizados en UCI pediátrica, la VNI mejoró significativamente su intercambio gaseoso, reduciendo significativamente la frecuencia respiratoria dentro de la primera hora de tratamiento9. Sólo 3 de los 28 pacientes requirieron de intubación y conexión a ventilador. En los pacientes con insuficiencia respiratoria aguda hipoxémica, el uso de VNI es adecuado, pero su eficacia debe ser evaluada dentro de las

* Servicio de Pediatría, Universidad del Desarrollo, Clínica Alemana, Santiago, Chile. 
primeras dos horas de implementado el procedimiento, con el objetivo de evitar una intubación $\operatorname{tardía}^{6,9,10}$. En los pacientes con hipercapnia, la reducción de la $\mathrm{PaCO}_{2}$ y frecuencia respiratoria después de dos horas de tratamiento con VNI es predictor de éxito terapéutico ${ }^{10}$. La VNI no está recomendada como primera opción en pacientes con síndrome de distress respiratorio agudo $^{10}$.

En relación al equipamiento, la interface debe ser elegida de acuerdo a la edad y características morfológicas del niño. Las almohadillas nasales pueden ser utilizadas en lactantes menores (menos de $5 \mathrm{~kg}$ ). En niños mayores son preferibles las máscaras nasales o máscaras faciales, aunque existe poca variedad de máscaras disponibles para niños pequeños. En ellos, el empleo de sistemas con arnés o casco han sido utilizados satisfactoriamente $e^{6,7}$.

Es importante destacar que en condiciones de descompensación respiratoria aguda se requiere de entrenamiento específico y de monitorización continua para iniciar la VNI, por lo que se aconseja su implementación en las unidades de cuidado intensivo.

\section{Ventilación mecánica no invasiva en condiciones crónicas}

La indicación mejor aceptada de VNI de larga duración es la insuficiencia respiratoria global diurna que es indicativa de hipoventilación alveolar crónica. También, los síntomas clínicos atribuibles a hipoventilación nocturna tales como sueño fraccionado, hipersomnolencia diurna, fatiga excesiva y cefalea matinal son importantes para decidir la indicación de VNI prolongada. Se recomienda en este caso confirmar la hipoventilación nocturna con una polisomnografía nocturna, pero no existe un criterio validado para el inicio de VNI en niños. Adicionalmente, una vez decidida la VNI en niños, es muy importante evaluar sus efectos en la $\mathrm{PaCO}_{2}$ diurna, calidad del sueño, crecimiento y desarrollo neuropsicológico, como estado de alerta, atención, concentración y comportamiento.

Guías clínicas de inicio de VNI están disponibles sólo para pacientes con miopatía de Duchenne y otras enfermedades neuromusculares $^{11,12}$. La hipercapnia diurna es un criterio clásico para implementar el procedimiento, pero la iniciación de VNI con la sola existencia de hipercapnia nocturna puede ser una alternativa valiosa a considerar, como ha sido recientemente comunicado ${ }^{13}$.
No existen guías clínicas o recomendaciones para pacientes con apnea obstructiva del sueño y con fibrosis quística, en ambos grupos de pacientes, la hipercapnia diurna es considerada una indicación de VNI. En ausencia de hipercapnia diurna, se recomienda efectuar un estudio de sueño en el caso de retraso en el crecimiento o fatiga excesiva, debido a que los síntomas de hipoventilación nocturna pueden ser sutiles, especialmente en lactantes.

La experiencia en VNI domiciliaria en niños en Francia fue reportada por Fauroux y cols. el 2003, quienes realizaron un estudio anónimo nacional cruzado utilizando un cuestionario enviado a todos los centros especializados que realizan VNI domiciliaria en casos de falla respiratoria crónica ${ }^{14}$. Se incluyeron todos los pacientes menores de 18 años que reciben VNI domiciliaria prolongada. Se obtuvo información detallada de 102 pacientes de 15 centros; cuatro de ellos atienden el $84 \%$ de los pacientes. El 7\% de los pacientes eran menores de 3 años, 35\% entre 411 años y 58\% mayores de 12 años. Los principales diagnósticos fueron enfermedades neuromusculares (34\%), apnea obstructiva y/o malformaciones craneofaciales (30\%), fibrosis quística (17\%), hipoventilación congénita (9\%), escoliosis $(8 \%)$ y otras alteraciones $(2 \%)$. La indicación de VNI fue hipoventilación nocturna (67\%), descompensación aguda $(28 \%)$ y retraso en el crecimiento $(21 \%)$. Los autores destacan el relativamente bajo número de pacientes pediátricos que reciben VNI domiciliaria en un país con gran experiencia en este procedimiento terapéutico, lo que apoya la creencia de que es subutilizada en este grupo etáreo.

El mayor beneficio de la VNI es la mejoría en la sobrevida, aunque esto sólo se ha demostrado en pacientes con enfermedad neuromuscular. El uso prolongado de VNI se ha asociado a una mejor sobrevida en los pacientes con distrofia muscular de Duchenne en un estudio desarrollado en Dinamarca ${ }^{15}$. No se ha demostrado mejor sobrevida en pacientes con enfermedad pulmonar crónica como fibrosis quística.

Los beneficios a largo plazo de la VNI deben incluir un efecto positivo sobre los desórdenes respiratorios del sueño, función pulmonar e intercambio gaseoso, función de la musculatura respiratoria, calidad de vida y particularmente en los niños interesa conocer los efectos beneficiosos sobre el crecimiento pulmonar y estado nutritivo. Estos efectos a largo plazo no han sido documentados en pacientes pediátricos, con la excepción de la mejoría de los trastornos respiratorios del sueño, función pulmonar e inter- 
cambio gaseoso, función de la musculatura respiratoria y calidad de vida en niños con enfermedades neuromusculares ${ }^{8,16,17}$.

El desafío a futuro para los pediatras que realizan VNI prolongada es demostrar los efectos benéficos del procedimiento en este grupo etáreo. Se plantea entonces como un desafío a futuro el demostrar la utilidad de la VNI prolongada en los niños con insuficiencia respiratoria crónica e hipercapnia.

\section{Bibliografía}

1.- PIRRET A M, SHERRING C L, TAI J A, GALBRAITH $\mathrm{N}$ E, PATEL R, SKINNER S M. Local experience with the use of nasal bubble CPAP in infants with bronchiolitis admitted to a combined adult/paediatric intensive care unit. Intensive Crit Care Nurs 2005; 21: 314-9.

2.- MARTINÓN-TORRES F, RODRÍGUEZ-NUÑEZ A, MARTINÓN-SÁNCHEZ J M. Nasal continuous positive airway pressure with heliox in infants with acute bronchiolitis. Respir Med 2006; 100: 1458-62.

3.- ESSOURI S, NICOT F, CLÉMENT A, GARABEDIAN E N, ROGER G, LOFASO F, et al. Noninvasive positive pressure ventilation in infants with upper airway obstruction: comparison of continuous and bilevel positive pressure. Intensive Care Med 2005; 31: 57480.

4.- FAUROUX B, PIGEOT J, POLKEY M I, ISABEY D, CLÉMENT A, LOFASO F. In vivo physiologic comparison of two ventilators used for domiciliary ventilation in children with cystic fibrosis. Crit Care Med 2001; 29: 2097-105.

5.- FAUROUX B, LOUIS B, HART N, ESSOURI S, LEROUX K, CLÉMENT A, et al. The effect of backup rate during non-invasive ventilation in young patients with cystic fibrosis. Intensive Care Med 2004; 30: 673-81.

6.- PIASTRA M, ANTONELLI M, CHIARETTI A, POLIDORI G, POLIDORI L, CONTI G. Treatment of acute respiratory failure by helmet-delivered noninvasive pressure support ventilation in children with acute leukemia: a pilot study. Intensive Care Med 2004; 30: 472-6.

7.- PIASTRA M, ANTONELli M, CARESTA E,
CHIARETTI A, POLIDORI G, CONTI G. Noninvasive ventilation in childhood acute neuromuscular respiratory failure: a pilot study. Respiration 2006; 73: 791-8.

8.- SIMONDS A K, WARD S, HEATHER S, BUSH A, MUNTONI F. Outcome of paediatric domiciliary mask ventilation in neuromuscular and skeletal disease. Eur Respir J 2000; 16: 476-81.

9.- FORTENBERRY J D, DEL TORO J, JEFFERSON L S, EVEY L, HAASE D. Management of pediatric acute hypoxemic respiratory insufficiency with bilevel positive pressure (BiPAP) nasal mask ventilation. Chest 1995; 108: 1059-64.

10.- ESSOURI S, CHEVRET L, DURAND P, HAAS V, FAUROUX B, DEVICTOR D. Noninvasive positive pressure ventilation: five years of experience in a pediatric intensive care unit. Pediatr Crit Care Med 2006; 7: 329-34.

11.- ROBERT D, WILLIG T N, LEGER P, PAULUS J. Long-term nasal ventilation in neuromuscular disorders: report of a consensus conference. Eur Respir J 1993; 6: 599-606.

12.- RUTGERS M, LUCASSEN H, KESTEREN R V, LEGER P. Respiratory insufficiency and ventilatory support. $39^{\text {th }}$ European Neuromuscular Centre International workshop. Neuromuscul Disord 1996; 6: 431-5.

13.- WARD S, CHATWIN M, HEATHER S, SIMONDS A K. Randomised controlled trial of non-invasive ventilation (NIV) for nocturnal hypoventilation in neuromuscular and chest wall disease patients with daytime normocapnia. Thorax 2005; 60: 1019-24.

14.- FAUROUX B, BOFFA C, DESGUERRE I, ESTOURNET B, TRANG H. Long-term noninvasive mechanical ventilation for children at home: A national survey. Pediatr Pulmonol 2003; 35: 119-25.

15.- JEPPESEN J, GREEN A, STEFFENSEN B F, RAHBEK J. The Duchenne muscular dystrophy population in Denmark, 1977-2001: prevalence, incidence and survival in relation to the introduction of ventilator use. Neuromuscul Disord 2003; 13: 804-12.

16.- SIMONDS A K, MUNTONI F, HEATHER S, FIELDING S. Impact of nasal ventilation on survival in hypercapnic Duchenne muscular dystrophy. Thorax 1998; 53: 949-52.

17.- MELLIES U, RAGETTE R, DOHNA SCHWAKE C, BOEHM H, VOIT T, TESCHLER H. Long-term noninvasive ventilation in children and adolescents with neuromuscular disorders. Eur Respir J 2003; 22: 631-6.

Correspondencia a:

Dr. Raúl J. Corrales V.

E-mail: rcorrales@alemana.cl 